\title{
Cost-effectiveness analysis of voice rehabilitation for patients with laryngeal cancer: a randomized controlled study
}

\author{
Mia Johansson $^{1,2}$ (D) $\cdot$ Caterina Finizia $^{3,4}$ (D) Josefine Persson ${ }^{5}$ (D) $\cdot$ Lisa Tuomi $^{3,4}$ (D)
}

Received: 20 December 2019 / Accepted: 13 February 2020 / Published online: 20 February 2020

(C) The Author(s) 2020

\begin{abstract}
Introduction Voice problems are common following radiotherapy for laryngeal cancer. Few studies exist covering the effect of voice rehabilitation, and no previous studies exist regarding the cost of said rehabilitation. This randomized controlled study aimed to analyze the cost-effectiveness of voice rehabilitation after radiotherapy for patients with laryngeal cancer.

Material and methods A total of 66 patients with laryngeal cancer with follow-up data 12 months post-radiotherapy were included. Patients were randomized into receiving either voice rehabilitation $(n=32)$ or no voice rehabilitation $(n=34)$. The patient outcome was measured as quality-adjusted life years (QALYs). The index range between 0 and 1 , where 0 equals death and 1 represents perfect health. The QALYs were assessed with the European Organization for Research and Treatment of Cancer questionnaire QLQ-C30 mapped to EuroQoL 5 Dimension values. The cost of rehabilitation and other healthcare visits was derived from hospital systems. The patients reported the total amount of sick leave days during the first 12 months following radiotherapy. The cost-effectiveness of the voice rehabilitation was compared with no rehabilitation intervention based on the incremental cost-effectiveness ratio.

Results The cost per gained QALY with voice rehabilitation compared to no rehabilitation from a societal perspective was $27,594 €(\mathrm{SEK}-250,852)$ which indicates that the voice rehabilitation is a cost-saving alternative compared to no rehabilitation due to lower costs and a slightly better health outcome. From a healthcare perspective, the voice rehabilitation indicates a cost $60,800 €$ (SEK 552,725) per gained QALY.

Conclusion From a societal perspective, i.e., including the costs of production loss, voice rehabilitation compared to no voice rehabilitation following radiotherapy for laryngeal cancer seems to be cost-saving. When analyzing only the healthcare costs in relation to health outcomes, voice rehabilitation indicates an incremental cost of $60,800 €$ per gained QALY, which is just above the threshold of the maximum willingness to pay level.
\end{abstract}

Keywords Laryngeal neoplasms $\cdot$ Voice therapy $\cdot$ Costs $\cdot$ Cost-effectiveness $\cdot$ Radiotherapy $\cdot$ Quality of life

Lisa Tuomi

lisa.tuomi@gu.se

1 Department of Oncology, Institute of Clinical Sciences, Sahlgrenska Academy at the Gothenburg University, Gothenburg, Sweden

2 Department of Oncology, Sahlgrenska University Hospital, Region Västra Götaland, Gothenburg, Sweden

3 Department of Otorhinolaryngology, Head and Neck surgery, Institute of Clinical Sciences, Sahlgrenska Academy, Gothenburg University, Gothenburg, Sweden

4 Department of Otorhinolaryngology, Sahlgrenska University Hospital, Region Västra Götaland, Gothenburg, Sweden

5 Health Economics and Policy, School of Public Health and Community Medicine, Institute of Medicine, University of Gothenburg, Gothenburg, Sweden

\section{Introduction}

Vocal dysfunction is a common side effect following radiotherapy for laryngeal cancer [1-3]. Studies show that voice problems occur in a majority of patients (40-100\%) and may persist up to 10 years following completion of radiotherapy [4-7]. The voice problems are often explained by reductions of the mucosal wave, scarring in the vocal fold tissue, inelasticity, and sometimes glottic inadequacy, which might result in compensatory behaviors in voice production $[1,3]$. The changes can affect the audible sound or lead to a feeling of troublesome voice production, both which may lead to a disrupted social life [8]. Therefore, voice rehabilitation has been suggested and assumed helpful for this patient group [9-11]. Few studies exist where the efficacy of voice 
rehabilitation for laryngeal cancer patients has been evaluated, but the studies that exist demonstrate a positive effect on voice quality, voice function, and health-related quality of life (HRQL) [5, 12-14]. Recently, our research group has reported the effects of voice rehabilitation in a randomized controlled study with follow-up up to 12 months following radiotherapy [15-20]. Voice rehabilitation improved health related quality of life, communicative function, and prevented the deterioration of voice quality over time, effects that remain 12 months post-radiotherapy. Even though positive effects have been demonstrated, no account has been made regarding the costs of said treatment. In order to implement the research results in clinical praxis, the cost of voice rehabilitation in relation to the patient's health effect need to be addressed. To the authors' knowledge, no studies assessing the cost-effectiveness of voice therapy exist. The objective of this study was therefore to analyze the cost-effectiveness of voice rehabilitation after radiotherapy for patients with laryngeal cancer.

\section{Material and methods}

\section{Participants}

All patients diagnosed with laryngeal cancer in the Region Västra Götaland are discussed on a weekly multidisciplinary tumor board, where treatment options are discussed and decided for each patient. Between the years 2000 and 2011, with an interruption of 2 years, the patients who were to receive curatively intended radiotherapy \pm chemotherapy were asked to participate in the study. Inclusion criteria were sufficient cognitive ability and sufficient knowledge of the Swedish language in order to fill out study questionnaires. Comorbidity was measured with the Adult Comorbidity Evaluation (ACE27) $[21,22]$.

\section{Study design}

The computerized randomization followed Pocock's sequential randomization method [23] for optimal allocation regarding age, gender, smoking habits, tumor site, tumor size, and patients' self-evaluation of communication [24]. Sample size was determined by an $80 \%$ power calculation, with dysphonia as the main variable. The groups were set to be of equal size. The power calculation prescribed a total of 80 patients, including an expected drop-out of ten patients. The voice rehabilitation group received voice rehabilitation after the completion of radiotherapy (between the 1-month and 6-month followup), while the control group received general vocal hygiene advice according to clinical practice. Both groups were followed up with audio recordings and questionnaires at similar time-points. The follow-ups were made at 1, 6, and 12 months post-radiotherapy.
Voice rehabilitation was given between 1 and 6 months post-radiotherapy, by speech-language pathologists. The rehabilitation protocol consisted of 10 occasions over the course of 10 weeks, approximately 30 min per rehabilitation session. The sessions included a specified set of exercises of breathing, relaxation, and phonation exercises, both indirect and direct therapy techniques. The patients were encouraged to exercise at home between sessions. The protocol has been described in detail elsewhere [18].

\section{Oncological treatment}

Radiotherapy was given in accordance with regional treatment guidelines, either as conventionally fractioned $(n=$ $45)$ or hyperfractioned $(n=21)$. Conventionally, fractionated therapy was given once daily in 2-2.4 Gy fractions to a total dose of 64.6-68 Gy. Hyperfractioned therapy entailed 1.7 Gy doses that were given twice daily to a total dose of $64.6 \mathrm{~Gy}$. Most patients with T2-T4 tumors also received irradiation to regional lymph nodes to a total dose of 40.8-46 Gy. Three patients received chemotherapy in addition to radiotherapy.

\section{Quality-adjusted life years}

The patient outcome was measured as quality-adjusted life years (QALYs). QALY is a measurement that combines health-related quality of life (HRQL) and life expectancy in one index. The index range between 0 and 1 , where 0 equals death and 1 represents perfect health. Thus, 1 QALY corresponds to 1 year of perfect health [25]. Life expectancy is not affected by the voice rehabilitation, why any difference in the gained QALYs is due to an increase in HRQL, often called QALY-weights. The QALYweights weres assessed with the European Organization for Research and Treatment of Cancer (EORTC) questionnaire QLQ-C30 mapped to EuroQoL 5 Dimensions (EQ5D) values by Proskorovsky et al. [26]. The EORTC QLQ-30 is a validated 30-item questionnaire [27]. The questionnaire includes five functional scales (Physical, Role, Cognitive, Emotional, and Social Functioning), three symptom scales (Fatigue, Pain, and Nausea/ Vomiting), a Global Health/HRQL scale, and six single items (Constipation, Diarrhea, Insomnia, Dyspnea, Appetite Loss, and Financial Difficulties). EQ-5D is a self-administered questionnaire consisting of five dimensions (Mobility, Self-Care, Pain, Usual Activities, and Anxiety/Depression) with three levels in each dimension (none, moderate and severe problems). In the mapping algorithm by Proskorovsky et al. [26], the EQ-5D health states were converted into a single health index using the UK value sets [28]. In the base case analysis, the full algorithm model by Proskorovsky et al. [26] was used, 
i.e., all the items in the EORTC QLQ-C30 questionnaire were used to map the EQ-5D QALY-weights. The EORTC QLQ-C30 was assessed 1, 6, and 12 months after radiotherapy. QALYs were estimated individually for each patient as the area under the curve by using time-weighted QALY-weights for the time spent in each health state [25]. Thus, the QALYs were estimated reflecting the change during the period between 1,6 , and 12 months post-radiotherapy. The total QALYs for each alternative were estimated as the means of the time-weighted individual QALYs.

\section{Costs}

The costs were categorized as either direct healthcare costs or loss of production. The cost of cancer treatment is not included in the analysis. All costs regarding hospital admission days and hospital visits over the 12 months following radiotherapy were derived from the hospital charts and hospital administration and were calculated for each patient. The loss of production was based on the number of sick leave days after radiotherapy treatment and during 1-year follow-up. The loss of production was valued by the human capital approach assuming that production loss is valued at market price [29], i.e., gross salaries and payroll taxes. A daily estimation including payroll taxes of $215 €$ (SEK 1950) [30] was used. The cost of sick leave was calculated for the able-bodied patients, i.e., the patients that were working at the time of the study $(n=26)$, where the patients were asked about the amount of sick leave during the 12 months following radiotherapy.

Costs are presented in euros (€/EUR) and in Swedish kronor (SEK) using 2013 exchange rates for conversion to Swedish kronor (SEK $8.98=1$ EUR).

\section{Cost-effectiveness analysis}

The cost-effectiveness of the voice rehabilitation was compared with no rehabilitation intervention based on the incremental cost-effectiveness ratio (ICER). The ICER is defined by the difference in cost between the two alternatives, divided by the difference in their effects. Thus, the ICER could be interpreted as the incremental cost associated with 1 additional QALY. The ICER is calculated as follows:

$\mathrm{ICER}=\frac{\text { Cost }_{\text {voice rehabilitation }}-\text { Cost }_{\text {no rehabilitation }}}{\mathrm{QALYs}_{\text {voice rehabilitation }}-\mathrm{QALYs}_{\text {no rehabilitation }}}$

The cost-effectiveness analyses are presented from two perspectives, the societal perspective (including both direct healthcare costs and loss of production), and the healthcare perspective (including direct healthcare costs). The costs and health outcomes were not discounted due to a 1-year time horizon.

\section{Assessing uncertainties}

A non-parametric bootstrapping was conducted to demonstrate the uncertainties surrounding the ICER. The bootstrapping was conducted using 1000 bootstrap replicates. The results of the bootstrapping are shown using a cost-effectiveness plane in Fig. 1. The cost-effectiveness plane graphically plots the differences in costs and QALYs between voice rehabilitation compared to no rehabilitation. No rehabilitation is plotted at the origin of the graph, and the incremental costs and QALYs with voice rehabilitation are represented at the $x$ - and $y$-axes. The maximum willingness to pay was set to $55,000 €$ (SEK 500,000) according to the Swedish National Board of Health and Welfare [31].

There is no existing algorithm for mapping EORTC QLQC30 to EQ-5D values for patients with laryngeal cancer; thus, a deterministic sensitivity analysis was carried out with the existing algorithms for other types of cancer [32-35].

\section{Statistical analysis}

The variable distribution was presented as mean and standard deviation $(95 \% \mathrm{CI})$ for continuous variables and as number and percentage for categorical variables. All significance tests were two-sided and conducted at the 5\% significance level. For comparison between groups, the non-parametric MannWhitney $U$ test was used due to skewed data. All the analyses were carried out in SPSS software (version 25, SPSS, Inc., Chicago, IL, USA).

\section{Results}

A total of 194 patients were assessed for eligibility in the study, and 163 patients met the inclusion criteria. Of these, 89 patients $(55 \%)$ chose to participate in the study and were allocated to voice rehabilitation group or control group. Twelve patients discontinued their participation before their first follow-up, leaving a total of 77 patients (87\%). An additional 11 patients discontinued their participation before the 12-month follow-up, leaving 32 patients in the voice rehabilitation group and 34 in the control group. Reasons for discontinuation were missed appointment $(n=2)$, laryngectomy $(n=4)$, and patient choice $(n=5)$.

The patient characteristics of included patients are listed in Table 1 . No statistically significant changes were found between the groups regarding age, sex, tumor location, tumor stage, comorbidity, or type of treatment. A total of 26 patients (39\%) were considered able-bodied, i.e., were working at the time of the study, and included in the analysis of the societal perspective. 

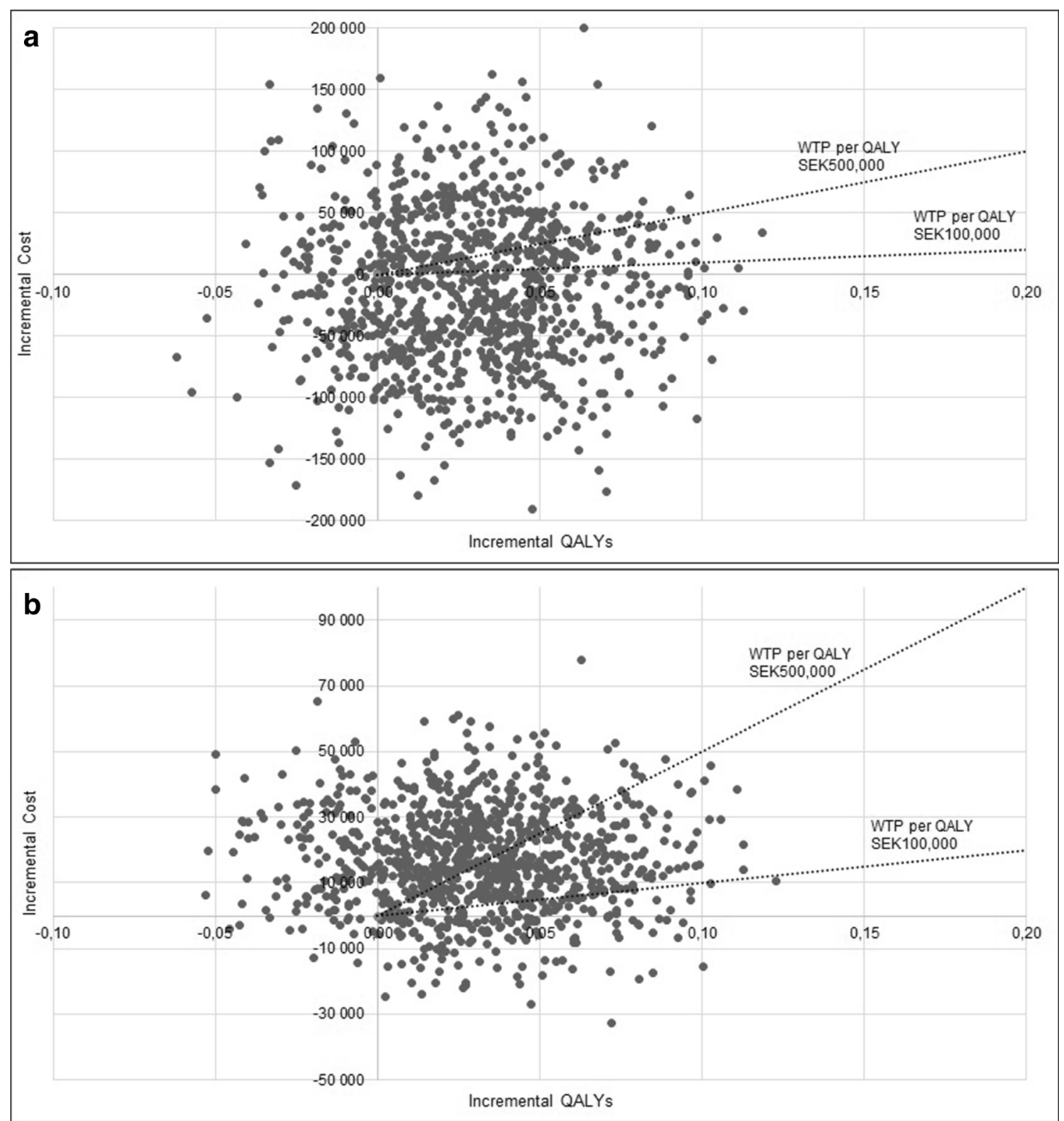

Fig. 1 Cost-effectiveness plane $\mathbf{a}$ in a societal perspective and $\mathbf{b}$ in a healthcare perspective

Table 2 shows the mean number of visits to oncologists and otorhinolaryngologists, hospital admission days, and costs. The mean healthcare cost for the group with voice rehabilitation was $6775 €$ ranging from $2893 €$ to 41,802 $€$ (SEK 61,594; range SEK 26,298 to SEK 380,017) and the mean healthcare cost for the group with no rehabilitation was $4945 €$ range from $453 €$ to $32,842 €$ (SEK 44,959; range SEK 4118 to SEK 298,868). There was a significant difference in the direct healthcare costs between the voice rehabilitation group and no rehabilitation group.

The mean cost due to loss of production for the group with voice rehabilitation was $14,512 €$, with a range from $0 €$ to $72,072 €$ (SEK 131,930; range SEK 0 to SEK 655,200) and the mean healthcare cost for the group with no rehabilitation was $17,173 €$ with a range from $0 €$ to $65,422 €$ (SEK 156,115 ; range SEK 0 to SEK 594,750).

The QALYs were calculated as the area under the curve by using time-weighted QALY-weight taking account of the time spent in each health state (Table 3). The mean QALYs were 0.87 (range 0.53 to 1 ) and 0.84 (range 0.57 to 1 ) in the voice rehabilitation and no rehabilitation group, respectively, indicating a difference in QALYs of 0.03 points. The difference in health outcomes was not significant.

The cost-effectiveness results are shown in Table 4. From a societal perspective, the cost per gained QALY with voice rehabilitation compared to no rehabilitation was $-27,594 €$ (SEK - 250,852). Thus, the voice rehabilitation is a cost- 
Table 1 Patient characteristics

\begin{tabular}{|c|c|c|c|}
\hline & Voice rehabilitation $n=32$ & No voice rehabilitation $n=34$ & $p$ value \\
\hline \multirow[t]{2}{*}{ Mean age (SD) } & $64.8(13.0)$ & $62.5(10.1)$ & ns \\
\hline & n $(\%)$ & n $(\%)$ & \\
\hline Sex & & & ns \\
\hline Male & $28(87.5)$ & $30(88)$ & \\
\hline Female & $4(12.5)$ & $4(12)$ & \\
\hline Smoking habits 1 year post-radiotherapy & & & ns \\
\hline Smoker & $6(19)$ & $2(6)$ & \\
\hline Non-smoker & $7(22)$ & $9(26)$ & \\
\hline Quit smoking $>12$ months ago & $19(59)$ & $23(68)$ & \\
\hline Tumor location & & & ns \\
\hline Glottis & $26(81)$ & $25(73.5)$ & \\
\hline Supraglottis & $6(19)$ & $8(23.5)$ & \\
\hline Subglottis & 0 & $1(3)$ & \\
\hline Tumor stage & & & ns \\
\hline 0 & $0(0)$ & $1(3)$ & \\
\hline I & $22(69)$ & $17(50)$ & \\
\hline II & $8(25)$ & $11(32)$ & \\
\hline III & $1(3)$ & $5(15)$ & \\
\hline IV & $1(3)$ & $0(0)$ & \\
\hline Comorbidity 1 year post-radiotherapy (ACE-27) & & & ns \\
\hline None & $14(44)$ & $14(41)$ & \\
\hline Mild & $9(28)$ & $15(44)$ & \\
\hline Moderate & $9(28)$ & $4(12)$ & \\
\hline Severe & $0(0)$ & $1(3)$ & \\
\hline Radiotherapy & & & ns \\
\hline Conventional & $23(72)$ & $22(65)$ & \\
\hline Hyperfractioned & $9(28)$ & $12(35)$ & \\
\hline Chemotherapy & $2(6)$ & $1(3)$ & ns \\
\hline
\end{tabular}

ACE-27 Adult Comorbidity Evaluation

saving alternative compared to no rehabilitation due to lower costs and associated with a slightly better health outcome, and thus a dominant alternative. From a healthcare perspective, the voice rehabilitation indicates a cost of $60,800 €$ (SEK $552,725)$ per gained QALY, which is just above the threshold of the maximum willingness to pay level.

\section{Sample uncertainty and sensitivity analysis}

A non-parametric bootstrapping with 1000 bootstrap replicates was conducted to assess the uncertainty in the costeffectiveness ratio. The results are presented in a costeffectiveness plane (Fig. 1), which graphically plots the differences in costs and QALYs between voice rehabilitation compared to no rehabilitation.

From a societal perspective and at a threshold of $55,000 €($ SEK 500,000$)$, there is a $66 \%$ probability that voice rehabilitation is cost-effective compare to no rehabilitation. From a healthcare perspective and at a threshold of $55,000 €($ SEK 500,000$)$, there is a $62 \%$ probability that voice rehabilitation is cost-effective compared to no rehabilitation.

Table 5 shows the sample's QALY weights using the different mapping algorithms indicating a difference in gained QALYs that ranges from 0.003 with the algorithm for breast and colorectal cancer to 0.030 with the algorithm for multiple myeloma. Thus, the ICER ranges from $61,500 €$ to $556,000 €$ (SEK 552,725 to SEK 5.1 million) when applying the different mapping algorithms.

\section{Discussion}

This study aimed to investigate the cost-effectiveness of a structured voice rehabilitation protocol following radiotherapy for laryngeal cancer. Previous studies have shown that the 
Table 2 Costs per patient during first year after radiotherapy presented in EUR (SD)

\begin{tabular}{|c|c|c|c|c|}
\hline & Total $(n=66)$ & Voice rehabilitation $(n=32)$ & No rehabilitation $(n=34)$ & $p$ value \\
\hline Number of visits to oncologists & $3.11(0.98)$ & $3.09(0.76)$ & $3.12(1.15)$ & n.s. \\
\hline Costs of visits to oncologists & $712(224)$ & $709(178)$ & $715(263)$ & n.s. \\
\hline Number of hospital admission days to Oncology & $0.64(2.58)$ & $0.59(3.31)$ & $0.68(1.59)$ & n.s. \\
\hline Costs of hospital admission to Oncology & $509(2066)$ & 475 (2687) & $541(1272)$ & n.s. \\
\hline Number of visits to otorhinolaryngologists & $1.79(1.93)$ & $1.81(2.40)$ & $1.76(1.37)$ & n.s. \\
\hline Costs of visits to otorhinolaryngologists & $493(531)$ & $500(662)$ & $486(378)$ & n.s. \\
\hline Number of hospital admission days to otorhinolaryngology & $4.06(9.64)$ & $3.72(9.72)$ & $4.38(9.70)$ & n.s. \\
\hline Cost of hospital admission to otorhinolaryngology & $2937(6970)$ & $2690(7027)$ & $3170(7012)$ & n.s. \\
\hline Number of other healthcare visits & $0.23(1.08)$ & $0.06(0.35)$ & $0.38(1.46)$ & n.s. \\
\hline Costs of other healthcare visits & $56(266)$ & $15(87)$ & $94(360)$ & n.s. \\
\hline Cost of voice rehabilitation & 2470 & 2470 & - & - \\
\hline Total direct healthcare costs & $5905(7364)$ & $6859(7726)$ & $5007(7002)$ & $<0.001$ \\
\hline Number of sick leave days & $78.82(111.36)$ & $67.66(107.44)$ & $85.72(116.01)$ & n.s. \\
\hline Loss of production & $16,079(23,784)$ & $14,692(23,330)$ & $17,385(24,479)$ & n.s. \\
\hline Total costs & $21,984(23,843)$ & $21,551(27,472)$ & $22,391(26,658)$ & n.s. \\
\hline
\end{tabular}

effects of voice rehabilitation include improved HRQL, which prevents a deterioration of perceived roughness and better self-perceived communicative function $[17,18,20]$. The present study showed that from a societal perspective, the voice rehabilitation is a cost-saving alternative compared to no rehabilitation due to lower costs and associated with a slightly better health outcome.

The gain from a societal perspective was the decreased number of sick leave days from an average of 85.72 with no rehabilitation to 67.66 with voice rehabilitation. The number of sick leave days in the group without voice rehabilitation is similar to a study by Cohen et al., where laryngeal cancer patients had a mean of 97.89 sick leave days over a 12month period [36]. Although, due to the wide range in sick leave days in the study population, the difference in production loss between the two groups was not statistically significant. The wide range in the production loss is also the main factor for the sampling uncertainty, illustrated in Fig. 1a. The result from a healthcare perspective was $60,800 €$ with voice rehabilitation compared to no rehabilitation. This result was just above the threshold of 55,000 $€$ set as a "rule-of-thumb" by the Swedish National Board of Health and Welfare, with $62 \%$ probability that the voice rehabilitation is cost-effective.

As previously stated, no mapping algorithm exists to convert EORTC QLQ-C30 data to utility scores for this patient group. The authors therefore looked at mapping algorithms for different patient groups, and the main algorithm chosen was the full model by Proskorovsky et al. [26]. This model was chosen since the age of the included patients as well as the EORTC QLQ-C30 values were similar to the present patient group. However, the prognosis for laryngeal cancer is generally better compared to the tumors stated in Table 5, which hinders comparison. Therefore, in order to perform further analyses, a mapping algorithm for the head and neck cancer population should be developed. Due to the lack of a mapping algorithm developed for this patient group, a sensitivity analysis performing the calculations with different algorithms was performed. This showed that the utility scores differed depending on which algorithm was used, subsequently affecting the ICER, which differed greatly (range from $61,500 €$ to $556,000 €$ in a healthcare perspective). Doble et al. 2016 [37] has assessed the external validity of the algorithms used

Table 3 Health outcomes based on the EORTC QLQ-C30 questionnaire mapped into EQ-5D QALY-weights (SD), comparisons between groups at each time-point, and mean time-weighted QALYs (95\% CI)

\begin{tabular}{lllll}
\hline & Total $(n=66)$ & Voice rehabilitation $(n=32)$ & No rehabilitation $(n=34)$ & $p$ value \\
\hline 1 month after radiotherapy & $0.80(0.15)$ & $0.81(0.14)$ & $0.79(0.17)$ & $0.86(0.14)$ \\
6 months after radiotherapy & $0.88(0.13)$ & $0.89(0.13)$ & $0.86(0.14)$ & n.s. \\
12 months after radiotherapy & $0.88(0.14)$ & $0.90(0.14)$ & $0.84(0.80-0.89)$ & n.s. \\
Mean time-weighted QALYs $(95 \%$ CI $)$ & $0.86(0.82-0.92)$ & $0.87(0.83-0.92)$ & n.s. \\
\hline
\end{tabular}

$Q A L Y$ quality-adjusted life year, $C I$ confidence interval 
Table 4 Cost-effectiveness results

Difference in QALYs $(95 \%$ CI $)$ Difference in costs (95\% CI) ICER EUR/QALY ICER SEK/QALY

Societal perspective

Voice rehabilitation vs no rehabilitation $0.03(-0.03-0.09)$

Healthcare perspective

Voice rehabilitation vs no rehabilitation $0.03(-0.03-0.09)$

$-7550(-130,380-113,598) \quad-27,594 \quad-250,852$

$16,635(-15,402-50,772) \quad 60,800 \quad 552,725$

QALY quality-adjusted life year, EUR euro, SEK Swedish kronor, ICER incremental cost effectiveness ratio, $C I$ confidence interval

in the deterministic sensitivity analysis resulted in a poor predictive accuracy. The result also indicated that the algorithms are insensitive to the grouping of tumor type and more sensitive to severity of the disease. Due to this, the authors recommend that an extensive scenario analysis should be conducted when the algorithms are used in cost-effectiveness analyses. The deterministic sensitivity analysis in this study confirms this statement resulting in a wide range of ICERs.

Additionally, the utility scores are mapped from the EORTC QLQ-C30, which might not be the most appropriate questionnaire to find the differences that are expected following voice rehabilitation. The EORTC QLQ-C30 is developed for measuring HRQL in cancer patients in general, and previously, the laryngeal cancer population has been found to demonstrate good HRQL at 1 year following treatment when using the EORTC QLQ-C30, with values comparable to the values of a normative population [38, 39]. However, when measured with tumor specific questionnaires, or questionnaires regarding communicative function, patients do report problems at 1 year following oncologic treatment [39, 40]. Therefore, the cost-effectiveness might be better calculated using another method, better corresponding to the changes that are expected to occur following voice rehabilitation, or should be completed with comparisons of effects in other domains such as communication and speech, for example, the speech domain of the EORTC QLQ head and neck module or the self-evaluation of communication experiences after laryngeal cancer [24, 40, 41].
The main strength with this cost-effectiveness analysis is that the data were collected prospective alongside a clinical trial and that the data are patient reported. A limitation to this study is the small patient sample with large variation of healthcare utilization and production loss, as well as that the power calculation for sample size was performed for voice outcomes only, and not costs and QALYs. Another limitation is that the sick leave days were derived mainly from the patients themselves, who were asked to state, in months, how much sick leave they had from work with increased risk of recall-bias. The number of sick leave day would probably be more accurate if this had been collected more precise, i.e., if the patient stated number of days or collected from registries. However, in this case, it was not possible, possibly leading to some bias regarding the total number of days of sick leave.

\section{Conclusion}

Voice rehabilitation compared to no voice rehabilitation following radiotherapy for laryngeal cancer seems to be cost-saving in a societal perspective, i.e., when including both healthcare costs and the costs of production loss. When analyzing only the healthcare costs in relation to quality outcomes, voice rehabilitation indicates a cost per QALY which is just above the maximum willingness to pay level. Despite this, this study provides important information to decision makers regarding the benefits of voice rehabilitation in a societal perspective.

Table 5 Health outcomes based on the EORTC QLQ-C30 questionnaire mapped into EQ-5D QALY-weights using different mapping algorithms

\begin{tabular}{|c|c|c|c|c|c|c|}
\hline Type of cancer & Total $(n=66)$ & Voice rehabilitation $(n=32)$ & No rehabilitation $(n=34)$ & Difference in QALYs & $p$ value & Source of algorithm \\
\hline Multiple myeloma & 0.86 & 0.87 & 0.84 & 0.03 & n.s. & [21] Full model* \\
\hline Multiple myeloma & 0.84 & 0.86 & 0.83 & 0.03 & n.s. & [21] Trimmed model \\
\hline Inoperable esophageal & 0.80 & 0.82 & 0.80 & 0.02 & n.s. & [32] Full model \\
\hline Gastric & 0.60 & 0.63 & 0.60 & 0.03 & n.s. & [26] EQ-5D \\
\hline Gastric & 0.69 & 0.70 & 0.69 & 0.01 & n.s. & [26] SF-6D \\
\hline Gastric & 0.73 & 0.74 & 0.72 & 0.02 & n.s. & {$[26] 15 \mathrm{D}$} \\
\hline Non-small cell lung & 0.82 & 0.82 & 0.81 & 0.01 & n.s. & [33] Full model \\
\hline Breast & 0.95 & 0.82 & 0.81 & 0.01 & n.s. & [24] Full model \\
\hline Breast and colorectal & 0.98 & 0.98 & 0.98 & 0.00 & n.s. & [25] Full model \\
\hline
\end{tabular}

*Base case analysis 
Author contributions All authors contributed to the study conception and design. Material preparation and data collection were performed by authors Caterina Finizia, Mia Johansson, and Lisa Tuomi. Analysis was performed by Josefine Persson. The first draft of the manuscript was written by Lisa Tuomi and Josefine Persson, and all authors commented on previous versions of the manuscript. All authors read and approved the final manuscript

Funding information Open Access funding provided by University of Gothenburg. This study was funded by grants from the Assar Gabrielsson foundation, Lions Cancer foundation West, the Swedish Cancer Society, the Research and Development Council (FoU) in Region Västra Götaland, the Sahlgrenska University Hospital, and the Medical Faculty of Gothenburg University, Sweden.

\section{Compliance with ethical standards}

The study was conducted in accordance with the Declaration of Helsinki and was approved by the regional ethical committee. Before inclusion in the study, all participants gave their informed consent.

Conflict of interest The authors declare that they have no conflict of interest.

Open Access This article is licensed under a Creative Commons Attribution 4.0 International License, which permits use, sharing, adaptation, distribution and reproduction in any medium or format, as long as you give appropriate credit to the original author(s) and the source, provide a link to the Creative Commons licence, and indicate if changes were made. The images or other third party material in this article are included in the article's Creative Commons licence, unless indicated otherwise in a credit line to the material. If material is not included in the article's Creative Commons licence and your intended use is not permitted by statutory regulation or exceeds the permitted use, you will need to obtain permission directly from the copyright holder. To view a copy of this licence, visit http://creativecommons.org/licenses/by/4.0/.

\section{References}

1. Bibby JR, Cotton SM, Perry A et al (2008) Voice outcomes after radiotherapy treatment for early glottic cancer: assessment using multidimensional tools. Head Neck 30(5):600-610

2. Agarwal JP, Baccher GK, Waghmare CM, Mallick I, GhoshLaskar S, Budrukkar A, Pai P, Chaturvedi P, D'Cruz A, Shrivastava SK, Dinshaw KA (2009) Factors affecting the quality of voice in the early glottic cancer treated with radiotherapy. Radiother Oncol 90(2):177-182

3. Krengli M, Policarpo M, Manfredda I, Aluffi P, Gambaro G, Panella M, Pia F (2004) Voice quality after treatment for T1a glottic carcinoma radiotherapy versus laser cordectomy. Acta Oncol 43(3):284-289

4. Morgan DA, Robinson HF, Marsh L, Bradley PJ (1988) Vocal quality 10 years after radiotherapy for early glottic cancer. Clin Radiol 39(3):295-296

5. van Gogh CD, Verdonck-de Leeuw IM, Boon-Kamma BA, Rinkel RN, de Bruin MD, Langendijk JA, Kuik DJ, Mahieu HF (2006) The efficacy of voice therapy in patients after treatment for early glottic carcinoma [randomized controlled trial research support, non-U.S. Gov't]. Cancer. 106(1):95-105

6. Hocevar-Boltezar I, Zargi M (2000) Voice quality after radiation therapy for early glottic cancer. Arch Otolaryngol Head Neck Surg 126(9):1097-1100
7. Rinkel RN, Verdonck-de Leeuw IM, van Reij EJ et al (2008) Speech Handicap Index in patients with oral and pharyngeal cancer: better understanding of patients' complaints. Head Neck 30(7): 868-874

8. Verdonck-de Leeuw IM, Keus RB, Hilgers FJ et al (1999) Consequences of voice impairment in daily life for patients following radiotherapy for early glottic cancer: voice quality, vocal function, and vocal performance. Int J Radiat Oncol Biol Phys 44(5): 1071-1078

9. Campos RJ, Maciel CT, Cesca MG et al (2011) Voice analysis after cancer treatment with organ preservation [evaluation studies]. Head Neck Oncol 3:19

10. Finizia C, Dotevall H, Lundstrom E et al (1999) Acoustic and perceptual evaluation of voice and speech quality: a study of patients with laryngeal cancer treated with laryngectomy vs irradiation. Arch Otolaryngol Head Neck Surg. 125(2):157-163

11. Hocevar-Boltezar I, Zargi M, Strojan P (2009) Risk factors for voice quality after radiotherapy for early glottic cancer. Radiother Oncol 93(3):524-529

12. Fex S, Henriksson B (1969) Phoniatric treatment combined with radiotherapy of laryngeal cancer for the avoidance of radiation damage. Acta Otolaryngol Suppl 263:128-129

13. Jotic A, Stankovic P, Jesic S, Milovanovic J, Stojanovic M, Djukic V (2012) Voice quality after treatment of early glottic carcinoma. J Voice 26(3):381-389

14. van Gogh CD, Verdonck-de Leeuw IM, Langendijk JA et al (2012) Long-term efficacy of voice therapy in patients with voice problems after treatment of early glottic cancer. J Voice 26(3):398-401

15. Bergstrom L, Ward EC, Finizia C (2016) Voice rehabilitation for laryngeal cancer patients: functional outcomes and patient perceptions. Laryngoscope 126(9):2029-2035

16. Bergstrom L, Ward EC, Finizia C (2017) Voice rehabilitation after laryngeal cancer: associated effects on psychological well-being. Support Care Cancer 25(9):2683-2690

17. Karlsson T, Johansson M, Andrell P et al (2015) Effects of voice rehabilitation on health-related quality of life, communication and voice in laryngeal cancer patients treated with radiotherapy: a randomised controlled trial. Acta Oncol 54(7):1017-1024

18. Tuomi L, Andréll P, Finizia C (2014) Effects of voice rehabilitation after radiation therapy for laryngeal cancer: a randomized controlled study. Int J Radiat Oncol Biol Phys 89(5):964-972

19. Tuomi L, Bjorkner E, Finizia C (2014) Voice outcome in patients treated for laryngeal cancer: efficacy of voice rehabilitation. J Voice 28(1):62-68

20. Karlsson T, Tuomi L, Andrell P et al (2017) Effects of voice rehabilitation after radiotherapy for laryngeal cancer: a longitudinal study. Logoped Phoniatr Vocol 42(4):167-177

21. Kaplan MH, Feinstein AR (1974) The importance of classifying initial co-morbidity in evaluating the outcome of diabetes mellitus. J Chronic Dis 27(7-8):387-404

22. Piccirillo JF (2000) Importance of comorbidity in head and neck cancer [comparative study]. Laryngoscope. 110(4):593-602

23. Pocock SJ, Simon R (1975) Sequential treatment assignment with balancing for prognostic factors in the controlled clinical trial. Biometrics. 31(1):103-115

24. Finizia C, Bergman B, Lindström J (1999) A cross-sectional validation study of self-evaluation of communication experiences after laryngeal Cancer: a questionnaire for use in the voice rehabilitation of laryngeal cancer patients. Acta Oncol 38(5):573-580

25. Glick HA, Doshi JA, Sonnad SS et al (2015) Economic evaluation in clinical trials, 2nd edn. Oxford University Press, Oxford

26. Proskorovsky I, Lewis P, Williams CD et al (2014) Mapping EORTC QLQ-C30 and QLQ-MY20 to EQ-5D in patients with multiple myeloma. Health Qual Life Outcomes 12(35):35

27. Aaronson NK, Ahmedzai S, Bergman B et al (1993) The European Organization for Research and Treatment of Cancer QLQ-C30: a 
quality-of-life instrument for use in international clinical trials in oncology [multicenter study]. J Natl Cancer Inst 85(5):365-376

28. Dolan P (1997) Modeling valuations for Euroqol health states. Med Care 35(11):1095-1108

29. Drummond M, Sculpher M, Torrance G et al (2015) Methods for the economic evaluation of health care programmes, 4rth edn. Oxford University Press, Oxford

30. Statistics Sweden. Average basic salary, monthly salary and women's salary as a percentage of men's salary by region, sector, occupation and sex. Year 2015. http://www.scb.se/2015 [cited 2018 June 18]

31. Socialstyrelsen. Nationella riktlinjer - metodbeskrivning. https:// www.socialstyrelsen.se/SiteCollectionDocuments/ metodbeskrivning-nationella-riktlinjer.pdf

32. Kim EJ, Ko SK, Kang HY (2012) Mapping the cancer-specific EORTC QLQ-C30 and EORTC QLQ-BR23 to the generic EQ5D in metastatic breast cancer patients. Qual Life Res 21(7): 1193-1203

33. Kim SH, Jo MW, Kim HJ et al (2012) Mapping EORTC QLQ-C30 onto EQ-5D for the assessment of cancer patients. Health Qual Life Outcomes 10:151

34. Kontodimopoulos N, Aletras VH, Paliouras D et al (2009) Mapping the cancer-specific EORTC QLQ-C30 to the preference-based EQ5D, SF-6D, and 15D instruments. Value Health 12(8):1151-1157
35. McKenzie L, van der Pol M (2009) Mapping the EORTC QLQ C30 onto the EQ-5D instrument: the potential to estimate QALYs without generic preference data. Value Health 12(1):167-171

36. Cohen SM, Kim J, Roy N, Asche C, Courey M (2012) The impact of laryngeal disorders on work-related dysfunction. Laryngoscope 122(7):1589-1594

37. Doble B, Lorgelly P (2016) Mapping the EORTC QLQ-C30 onto the EQ-5D-3L: assessing the external validity of existing mapping algorithms. Qual Life Res 25:891-991

38. Hammerlid E, Taft C (2001) Health-related quality of life in longterm head and neck cancer survivors: a comparison with general population norms. Br J Cancer 84(2):149-156

39. Nordgren M, Abendstein H, Jannert M et al (2003) Health-related quality of life five years after diagnosis of laryngeal carcinoma. Int $\mathrm{J}$ Radiat Oncol Biol Phys 56(5):1333-1343

40. Johansson M, Ryden A, Finizia C et al (2008) BMC Cancer 8:80

41. Bjordal K, Ahlner-Elmqvist M, Tollesson E, Jensen AB, Razavi D, Maher EJ, Kaasa S (1994) Development of a European Organization for Research and Treatment of Cancer (EORTC) questionnaire module to be used in quality of life assessments in head and neck cancer patients. EORTC quality of life study group [research support, non-U.S. Gov't]. Acta Oncol 33(8):879-885

Publisher's note Springer Nature remains neutral with regard to jurisdictional claims in published maps and institutional affiliations. 